\title{
Distinct Intracellular Calcium Transients in Neurites and Somata Integrate Neuronal Signals
}

\author{
Friedrich W. Johenning, ${ }^{1,3}$ Michal Zochowski, ${ }^{2}$ Stuart J. Conway, ${ }^{4}$ Andrew B. Holmes, ${ }^{4}$ Peter Koulen, ${ }^{1}$ and \\ Barbara E. Ehrlich ${ }^{1,2}$ \\ Departments of ${ }^{1}$ Pharmacology and ${ }^{2}$ Cellular and Molecular Physiology, Yale University, New Haven, Connecticut 06520, \\ ${ }^{3}$ Department of Neuroanatomy, University Hospital Eppendorf, 20246 Hamburg, Germany, and ${ }^{4}$ Department of \\ Chemistry, University of Cambridge, Cambridge CB2 1EW, United Kingdom
}

Intracellular calcium signals have distinct temporal and spatial patterns in neurons in which signal initiation and repetitive spiking occurs predominantly in the neurite. We investigated the functional implications of the coexpression of different isoforms of ryanodine receptors (RyR) and inositol 1,4,5trisphosphate receptors (InsP3Rs) using immunocytochemistry, Western blotting, and calcium imaging in neuronally differentiated PC12 cells. InsP3R type III, an isoform that has been shown to be upregulated in neuronal apoptosis, is exclusively expressed in the soma, serving as a gatekeeper for highmagnitude calcium surges. InsP3R type I is expressed throughout the cell and can be related to signal initiation and repetitive spiking in the neurite. RyR types 2 and 3 are distributed throughout the cell. In the soma, they serve as amplifying molecular switches, facilitating recruitment of the InsP3R type III-dependent pool. In the neurite, they decrease the probability of repetitive spiking. Use of a cell-permeant analog of InsP3 suggested that regional specificity in InsP3 production and surface-to-volume effects play minor roles in determining temporal and spatial calcium signaling patterns in neurons. Our findings suggest that additional modulatory processes acting on the intracellular channels are necessary to generate spatially specific calcium signaling.

Key words: intracellular calcium signaling; inositol 1,4,5 trisphosphate; InsP3 receptor; ryanodine receptor; PC12 cells; neurite; soma
Neurons use changes in intracellular free calcium for many important functions, including neurite outgrowth, gene expression, neurodegeneration, and neurotransmitter release. The concentration of intraneuronal calcium can be raised by voltage, ligandgated, or store-operated calcium channels on the plasma membrane (Ghosh and Greenberg, 1995). An alternative route is calcium release from internal stores mediated by two classes of intracellular calcium release channels, the ryanodine receptor (RyR) and the inositol 1,4,5-trisphosphate (InsP3) receptor (InsP3R) (Ehrlich et al., 1994; Rizzuto, 2001). The endoplasmic reticulum and its calcium signaling mechanisms form a membrane system that has been described as a "neuron-within-a-neuron," with all of the necessary components for signal integration, regenerative processes, conduction, and memory (Berridge, 1998).

The intracellular calcium release channels form a superfamily.

\footnotetext{
Received Dec. 13, 2001; revised Feb. 25, 2002; accepted March 8, 2002.

This work was supported by National Institutes of Health Grant GM63496 and a German National Merit Scholarship Foundation scholarship (F.W.J.). Antibodies for the ryanodine receptor were kindly provided by Dr. Vincenzo Sorrentino. InsP3R type II antibodies were a kind gift from Dr. Richard Wojcikiewicz. We thank the Biotechnology and Biological Sciences Research Council (United Kingdom) for financial support and the Engineering and Physical Sciences Research Council (United Kingdom) for provision of the Swansea Mass Spectrometry Service. We thank Drs. Martin Bootman and Peter Lipp for helpful discussions and access to InsP3-BM ester. Drs. E. Thrower, A. Sardini, and M. Nathanson made helpful comments on this manuscript, and B. DeGray provided excellent technical support.

Correspondence should be addressed to Barbara E. Ehrlich, Department of Pharmacology, Yale University, 333 Cedar Street, New Haven, CT 06520-8066. E-mail: barbara.ehrlich@yale.edu.

M. Zochowski's present address: Department of Physics, University of Michigan, Ann Arbor, MI 48109.

P. Koulen's present address: Department of Pharmacology and Neuroscience, University of North Texas Health Science Center, 3500 Camp Bowie Boulevard, Fort Worth, TX 76107-2699.

Copyright (C) 2002 Society for Neuroscience $\quad 0270-6474 / 02 / 225344-10 \$ 15.00 / 0$
}

There are three different RyR subtypes. Although the biophysical properties of the three isoforms are remarkably similar (Sitsapesan and Williams, 1998), the tissue specificity (Giannini et al., 1995), regulation by associated proteins (MacKrill, 1999), and phosphorylation (Marx et al., 2001) are isoform specific. The InsP3R also has three different subtypes, which differ in their biophysical properties (Perez et al., 1997; Hagar et al., 1998; Mak et al., 2000), their susceptibility to modulation by accessory proteins (Patel et al., 1999), and their subcellular localization in polarized cells (Hagar et al., 1998; Hirata et al., 1999, 2002). This degree of functional and molecular diversity has an impact on the cellular level. For example, differences in InsP3 dependence of the activation of calcium release for InsP3R type I and InsP3R type III have been reported when comparing permeabilized cell lines predominantly expressing one subtype (Wojcikiewicz and Luo, 1998).

The present work focuses on the mechanisms determining the initiation of regenerative calcium waves and the temporal and spatial patterns of global calcium signals, which are regenerative calcium transients spreading throughout the entire cell and have to be distinguished from elementary calcium signals that are confined to certain subcellular regions (Bootman et al., 2001). Several studies have discussed the initiation of calcium transients and waves in neurons or model cells. At the global level, InsP3 production via metabotropic glutamate receptor (mGluR) activation can mobilize intracellular calcium in dendrites of CA1 neurons and trigger regenerative calcium waves throughout the cell (Jaffe and Brown, 1994). Recently, it has been shown that these waves can also be triggered by repetitive synaptic activation of mGluRs, with a tendency to initiate at the proximal apical dendrite (Nakamura et al., 1999, 2000). The initiation site has been 
proposed to be a function of different surface receptor distributions in neuronally differentiated PC12 cells (Lorenzon et al., 1995). Other authors have suggested that differences in the surface-to-volume ratio between somata and neurites are responsible for local accumulation of InsP3 in neurites (Fink et al., 2000; van Acker et al., 2000). In HeLa cells, the initiation site of global calcium signals is perinuclear, but neither different plasma membrane receptor distributions nor different InsP3R isoforms were observed (Thomas et al., 2000), suggesting that additional factors are responsible for signal initiation.

Independent of the question how their initiation site is determined, these regenerative calcium waves appear to have a large impact on cellular function because they can transmit signals throughout the neuron and encode information using different temporal, spatial, and quantitative patterns (Berridge, 1998). Global calcium signals also have a carefully controlled activation threshold because they can contribute to both neuromodulatory and neurodegenerative processes by driving various intracellular signal transduction cascades (Usachev and Thayer, 1999). A key to understanding how the quantitative and spatiotemporal patterning of global calcium signals are controlled lies in the interaction of the aforementioned subtypes and classes of intracellular calcium release channels.

In this paper, we combine immunocytochemistry and Western blot analysis with calcium imaging to analyze the subcellular distribution and differential expression of InsP3R and RyR isoforms in a neuronally derived cell line and to examine kinetic differences between neuritic and somatic calcium signaling at the global level.

\section{MATERIALS AND METHODS}

Cell culture. PC12 cells were grown in DMEM high-glucose $(4.5 \mathrm{gm} / 1)$ medium supplemented with $10 \%$ horse serum, $5 \%$ fetal calf serum, 25 $\mathrm{U} / \mathrm{ml}$ penicillin, and $25 \mu \mathrm{g} / \mathrm{ml}$ streptomycin and cultured in a watersaturated atmosphere at $37^{\circ} \mathrm{C}$ and $5 \% \mathrm{CO}_{2}$. To induce differentiation, cells were plated onto poly-L-lysine-coated coverslips or flasks and treated with $100 \mathrm{ng} / \mathrm{ml}$ nerve growth factor (NGF). A dilution of 1:50 was used for cell plating so that the cells reached a confluency of $20 \%$ after differentiation with NGF for $7 \mathrm{~d}$.

Antibodies. Each InsP3 receptor isoform was detected using isoformspecific antibodies. InsP3R type I antibodies were affinity purified from a rabbit polyclonal antiserum directed against the $19 \mathrm{C}$-terminal residues of the mouse InsP3 receptor type I (Mignery et al., 1989) and were custom produced by Research Genetics (Huntsville, AL). InsP3R type II antibodies were affinity purified from a rabbit polyclonal antiserum directed against the $18 \mathrm{C}$-terminal residues of the rat InsP3R type II (Wojcikiewicz, 1995) and were kindly provided by Dr. Richard Wojcikiewicz (State University of New York, Syracuse, NY). A commercially available monoclonal antibody was used to label the N-terminal region of the human InsP3R type III (Hagar et al., 1998) (Transduction Laboratories, Lexington, K Y). Similarly, each RyR isoform was identified using isoform-specific antibodies (Giannini et al., 1995), all kindly provided by Dr. Vincenzo Sorrentino (Università degli di Siena, Siena, Italy). Commercially available antibodies for muscarinic acetylcholine receptors (mAChR) $\mathrm{M}_{1}$ and $\mathrm{M}_{5}$ [Research and Diagnostic Antibodies, Berkeley, CA (Ndoye et al., 1998)] and chromogranins A and B (ICN Pharmaceuticals, Costa Mesa, CA) were used. The secondary antibodies were Alexa 488 and 594 coupled to goat anti-rabbit, anti-mouse, or anti-rat IgG (Molecular Probes, Eugene, OR) and peroxidase conjugated to donkey anti-rabbit IgG (Vector Laboratories, Burlingame, CA) and goat antimouse (Bio-Rad, Hercules, CA).

Western blot analysis. PC12 cell homogenates were separated by SDS gel electrophoresis using 4-15\% polyacrylamide gels, and proteins were detected using standard Western blotting techniques (Hagar et al., 1998).

Immunocytochemistry. The cells were fixed using $4 \%$ paraformaldehyde [(PFA) w/v] in PBS (0.01 M), pH 7.4, for 20 min. Immunocytochemical labeling was performed using the indirect fluorescence method. Nonspecific binding sites were blocked by incubating the cells in PBS containing $0.05 \mathrm{M}$ glycine for $1 \mathrm{hr}$ and in PBS containing $10 \%$ normal goat serum, $1 \%$ bovine serum albumin, and $0.05 \%$ Triton X-100 (v/v) for 1 additional hour. Primary and secondary antibodies were diluted in PBS containing 3\% normal goat serum, $1 \%$ bovine serum albumin, and $0.05 \%$ Triton X-100. To label the mAChRs, cells were briefly permeabilized for 5 min with $0.05 \%$ Triton X-100. Triton X-100 was omitted from all of the solutions in the following steps. Moreover, a 1:1 mixture of $4 \%$ sucrose and $4 \%$ PFA in PBS was used for fixation when mAChRs were detected. Controls using the secondary antibodies showed only nonspecific background staining. For some images, the laser intensity and pinhole size had to be reduced to prevent saturation of the signal when compared with the control settings. The Zeiss (Oberkochen, Germany) LSM 510 system equipped with photomultipliers and a Zeiss Axiovert 100LM with a $63 \times$ plan apochromat oil immersion objective were used. Averaging four to six frames reduced noise. For excitation, an argon laser was used at 488 nm (Alexa 488) and $568 \mathrm{~nm}$ (Alexa 594). For detection of the signal, appropriate emission filters were used.(Alexa 488, 510 low-pass or 522/35 bandpass for double labeling; Alexa 594, $585 \mathrm{~nm}$ long-pass).

Calcium imaging. Confocal microscopy was used to measure intracellular calcium in PC12 cells. Cells grown on a glass coverslip were loaded with the fluorescent calcium dye Fluo-4. Cells were incubated at room temperature for 20 min with $5 \mu \mathrm{M}$ Fluo-4 AM in 20\% Pluronic F127 in DMSO and allowed an additional $20 \mathrm{~min}$ in dye-free media for deesterification. L15 medium was used as the extracellular solution. All experiments were performed using extracellular medium containing 5 mM EGTA to deplete extracellular calcium ("calcium-free solution"). The coverslip was used as the bottom of an open superfusion chamber The chamber was mounted onto the stage of a Zeiss Axiovert 135 inverted microscope. The cells were perfused continuously at $3 \mathrm{ml} / \mathrm{min}$. The chamber volume was $200 \mu$ l. Solution changes were accomplished rapidly by means of a valve attached to a four-chambered superfusion reservoir. Perfusion with calcium-free solution always started $1 \mathrm{~min}$ before addition of the muscarinic receptor agonist carbachol, which was applied for $2 \mathrm{~min}$. Responses at low agonist concentrations were only included in the analysis if cell viability could be confirmed by a response to a second pulse of $500 \mu \mathrm{M}$ carbachol 2 min after the perfusion with the low agonist concentration had ended. Cells were excited at $488 \mathrm{~nm}$, and the emission signal was detected with a $522 / 35 \mathrm{~nm}$ bandpass filter to avoid background fluorescence from dantrolene. Cells were observed using a $20 \times, 0.75$ numerical aperture objective, and whole-cell images were recorded at a rate of $5 \mathrm{~Hz}$. Increases in calcium were expressed as the ratio of fluorescence intensity of Fluo-4 over baseline $\left(F / F_{0}\right)$. The selfratio method $\left(F / F_{0}\right)$ was used because it is a measurement independent of factors such as dye concentration, excitation intensity, and detector efficiency (Hirata et al., 1998; Koizumi et al., 1999; Maravall et al., 2000; van Acker et al., 2000). The self-ratio could underestimate the intracellular free calcium concentration when large changes are measured. Fluo-4 has a high calcium affinity and a large dynamic range (Maravall et al., 2000), and thus the differences described are unlikely to surpass the dynamic range of the dye. If, however, the signals were in the nonlinear range, the magnitude of the signal would be underestimated, and therefore the differences would be even larger than reported. Background fluorescence was automatically subtracted from all measurements. There was no change in size, shape, or location of cells during the experiments. All experiments were performed at $21^{\circ} \mathrm{C}$.

Data analysis. The cells were divided into neuritic and somatic regions of interest (ROIs) over which the fluorescence intensity was measured Somatic and neuritic ROIs were approximately the same size $(5 \times 2 \mu \mathrm{m})$, and the whole cell was divided into at least eight different ROIs. The baseline of the signal was defined as the averaged fluorescence over the time interval before the fluorescence started to rise in the defined region of interest. The onset of the somatic and the neuritic signal was determined as the time point at which $F / F_{0}$ started to rise constantly above $10 \%$ of the interval between $F_{0}$ and $F_{\text {peak }}$ for the first time in a specific neuritic and somatic ROI after application of a 10-to-1 running average filter (see Fig. 3A). The flux rate was calculated as the slope of a line between the data point at which $F / F_{0}$ started to constantly rise above $10 \%$ of the interval between $F_{0}$ and $F_{\text {peak }}$ and the data point at which $F / F_{0}$ reached $90 \%$ of the interval between $F_{0}$ and $F_{\text {peak }}$. A response was defined as a repetitive spike when a decay and subsequent rise of $F / F_{0}$ of at least $60 \%$ of the difference between $F_{0}$ and $F_{\text {peak }}$ could be observed after application of a 10-to-1 running average filter.

Differences are called significant if $p<0.05$ using an unpaired Student's $t$ test, and all values are displayed as mean \pm SEM. 
A a

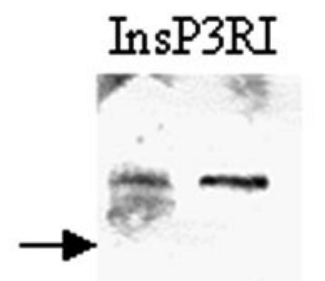

12

B a

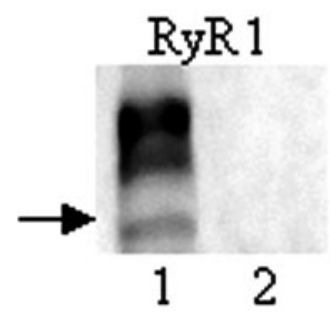

b InsP3R

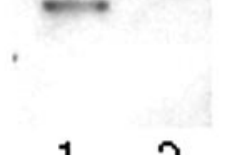

12

b

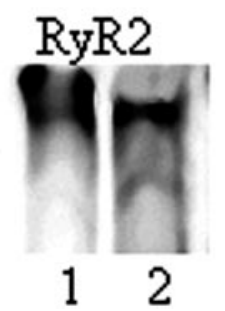

C
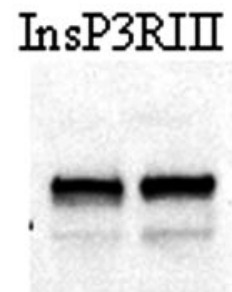

12

C

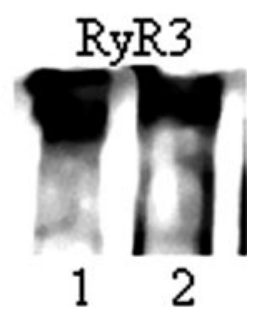

Figure 1. Differential InsP3R and RyR isoform expression determined by Western blot analysis. $A$ displays blots for different InsP3R isoforms, and $B$ shows the different RyR isoforms. $A a$, InsP3R type I, $10 \mu \mathrm{g}$ of mouse cerebellar microsomes in lane 1 and $100 \mu \mathrm{g}$ of PC12 cell homogenate in lane 2. $A b$, InsP3R type II, $50 \mu \mathrm{g}$ of liver homogenate in lane 1 and $200 \mu \mathrm{g}$ of PC12 cell homogenate in lane 2. Ac, InsP3R type III, $20 \mu \mathrm{g}$ of rat islet cell tumor cell microsomes in lane 1 and $100 \mu \mathrm{g}$ of PC12 cell lysate in lane 2. Ba, RyR type I, $10 \mu \mathrm{g}$ of mouse striated muscle microsomes in lane 1 and $200 \mu \mathrm{g}$ of PC12 cell lysate in lane 2. Bb, RyR type II, $25 \mu \mathrm{g}$ of canine cardiac muscle microsomes in lane 1 and $100 \mu \mathrm{g}$ of PC12 cell lysate lane 2. Bc, RyR type III, $100 \mu \mathrm{g}$ of mouse diaphragm homogenate in lane 1 and $100 \mu \mathrm{g}$ of PC12 cell lysate in lane 2. The arrows points to the position of the $200 \mathrm{kDa}$ molecular weight marker.

\section{RESULTS}

\section{Distribution of the different subtypes of InsP3Rs, RyRs, and mAChRs in NGF-treated PC12 cells}

Western blot analysis of PC12 cell homogenates confirmed the presence of the InsP3R subtypes I and III, whereas InsP3R type II was not detected (Fig. 1 $A$ ), despite using twice the amount of cell homogenate necessary to detect the other isoforms of the InsP3R. Two isoforms of the RyR, types 2 and 3, also were present in PC12 cell homogenates; RyR type 1 could not be detected (Fig. 1B).

The subcellular distribution of the intracellular calcium release channels found in PC12 cells was determined using immunocytochemistry with fluorescence-labeled secondary antibodies and confocal microscopy. Immunoreactivity for the InsP3R type I exhibited a diff use cytosolic staining pattern throughout the soma and neurite (Fig. 2Aa). The high signal intensity, indicating a nuclear localization of InsP3R type I in Figure 2, $A a$ and $A b$, might be explained, at least in part, by nonspecific binding of the polyclonal primary antibody for InsP3R type I to nuclear epitopes. For the InsP3R type III, a specific signal could only be detected in the somatic cytosol, suggesting that this protein is targeted to the somatic endoplasmic reticulum (Fig. 2Ac). When three-dimensional reconstructions of whole PC12 cells were examined using serial confocal sections, the absence of the InsP3R type III in the neurites was confirmed (data not shown). In addition, double labeling of the InsP3R types I and III confirms

the distribution pattern obtained using single-labeling experiments (Fig. 2Ae). The InsP3R type II was undetectable using identical immunocytochemical methods (data not shown). A specific signal of InsP3R type II antibody applying a similar immunocytochemical protocol has been shown in InsP3R type IIpositive hepatocytes and HepG2 cells (Hirata et al., 2002).

The two isoforms of the RyR identified by Western blot analysis, types 2 and 3 , were detected throughout the cytoplasm of the cell (Fig. 2Ba,Bb). Note that the signal for the RyR type 2 was most prominent in the somatic cytosol (Fig. $2 \mathrm{Ba}$ ). The RyR type 1 was not detectable using the same immunocytochemical methods (data not shown) but has been reported previously to detect RyR using a similar immunocytochemistry protocol (Giannini et al., 1995). The analysis of the distribution of mAChRs focused on the $\mathrm{M}_{1}$ and $\mathrm{M}_{5}$ subtypes (Fig. 2Ca,Cc). These subtypes are phospholipase $\mathrm{C}$ (PLC) coupled and are the predominant functional isoform coupled to activity of the G-protein $G_{q}$ in PC12 cells (Berkeley and Levey, 2000). Clusters of mAChRs were observed over the entire neurite. However, the majority of mAChR immunoreactivity was associated with the soma (Fig. $2 C a, C c)$. In Figure $2 D$, the predominantly neuritic staining for chromogranins A and B, high-capacity, low-affinity calcium binding proteins found predominantly in secretory vesicles, is displayed.

\section{Spatiotemporal patterns of intracellular calcium signals: correlation with agonist concentration and InsP3R subtype distribution}

The differential distribution of the InsP3R types I and III described above suggests a functional diversity of calcium signaling in spatially distinct regions of PC12 cells. Therefore, we next analyzed the spatiotemporal patterns of the intracellular calcium signals in the soma, which express InsP3R types I and III, and in neurites, which express InsP3R type I. The differential distribution implies functional differences because the InsP3R type $\mathrm{I}$ is activated at lower InsP3 concentrations than the InsP3R type III (Bezprozvanny et al., 1991; Hagar et al., 1998; Wojcikiewicz and Luo, 1998; Mak et al., 2000). In Figure 3, representative traces for experiments under different conditions are displayed. The $\mathrm{mAChR}$ agonist carbachol was applied for $2 \mathrm{~min}$. At $500 \mu \mathrm{M}$ carbachol (Fig. 3B) the temporal difference between the onset of the neuritic and the somatic signal is much smaller than at $50 \mu \mathrm{M}$ carbachol without (Fig. 3C) and with (Fig. 3D) the RyR antagonist dantrolene $(75 \mu \mathrm{M})$. The carbachol-induced calcium wave began in the neurites in $95 \%$ of the neurites analyzed $(n=83)$. The onset of the somatic and the neuritic signal was determined as described in Materials and Methods. At $500 \mu \mathrm{M}$ carbachol, there was an average delay of $1.7 \pm 0.5 \mathrm{sec}(n=13)$ between the first neuritic and somatic signals (Fig. $4 A$ ). Reducing the agonist concentration to $50 \mu \mathrm{M}$ resulted in a significant increase in the temporal delay to $3.4 \pm 0.6 \mathrm{sec}(n=19 ; p<0.05)$ (Fig. $4 A)$. A similar trend was seen in the presence of $75 \mu \mathrm{M}$ dantrolene; the delay was $2.1 \pm 0.6 \mathrm{sec}(n=12)$ at $500 \mu \mathrm{M}$ carbachol and $3.5 \pm$ $0.6 \mathrm{sec}$ at $50 \mu \mathrm{M}$ carbachol $(n=14 ; p<0.05)$ (Fig. $4 A$, white columns).

To quantify the degree of InsP3R activation at different agonist concentrations and to compare somatic and neuritic calcium signals, the flux rate was measured as $d\left(F / F_{0}\right) / d(t)$, as described in Materials and Methods. This parameter reflects the open probability of InsP3Rs during the main activation phase, as defined by a net efflux of calcium from the endoplasmic reticulum (Ogden and Capiod, 1997). In Figure 4B, the average of the flux rates in 
A

a

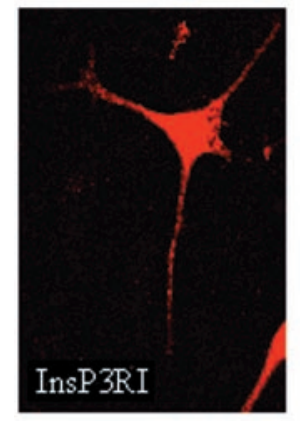

c

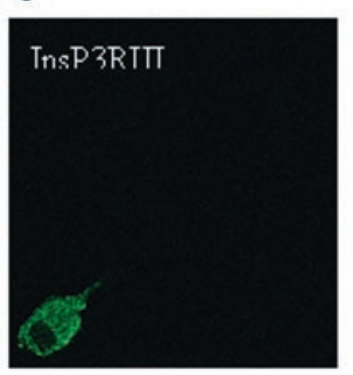

e

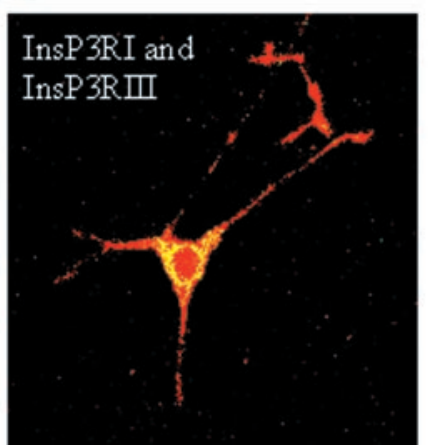

B

a

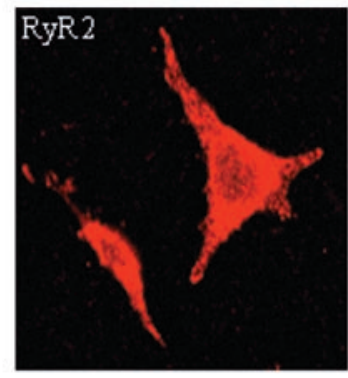

b

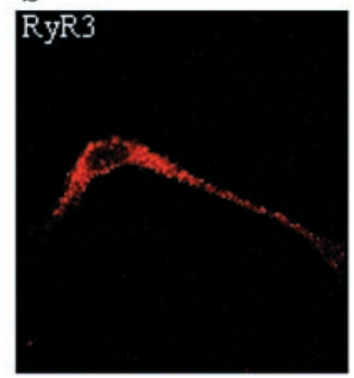

C

a

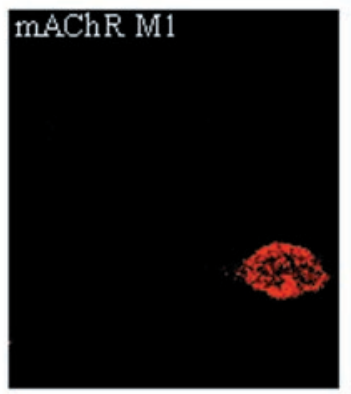

b

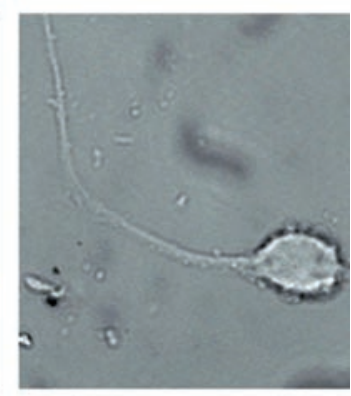

D

c
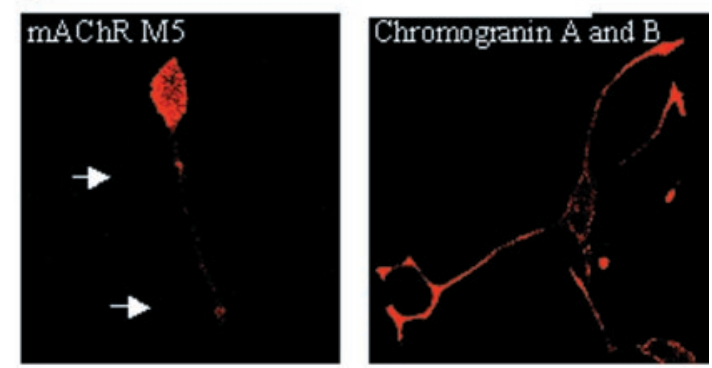

Figure 2. Subcellular distribution of InsP3R and RyR isoforms, mAChRs, and chromogranin A and B. InsP3R type I is found throughout the entire cytosol $(A a, A b)$. In $A c$, InsP3R type III immunoreactivity is found only in the somatic cytosol. $A d$ shows the cell in $A c$ as a differential interference contrast image. Taking different optical sections throughout the same cell, the absence of InsP3R type III immunoreactivity from the neurite could be confirmed. In $A e$, an image of a cell coimmunolabeled for InsP3R type I and III clearly displays the predominant somatic expression of InsP3R type III when compared with the ubiquitous expression of InsP3R type I. Colabeling for InsP3R type I and InsP3R type III is displayed in yellow, and exclusive expression of InsP3R type I is shown in red. Immunoreactivity for RyR type $2(B a)$ and type $3(B b)$ can be detected throughout the cytosol. RyR type 2 appears to be predominantly localized in the soma. $C a-C c$ shows that immunoreactivity for $\mathrm{M}_{1}$ and $\mathrm{M}_{5} \mathrm{mAChR}$ is predominantly localized to the soma. $\mathrm{Cb}$ is a differential interference contrast image of the same cell as in $\mathrm{Ca}$. In the neurites, only a few clusters of receptors can be observed ( $C c$, white arrows). $D$ shows that immunoreactivity for chromogranins A and B is predominantly localized to the neurites. There is only a weak subplasmalemmal staining pattern in the soma.

the neurite (black columns) were compared with the average of the flux rates in somata (gray columns) at three different agonist concentrations. At $500 \mu \mathrm{M}$ carbachol, both the somatic and neuritic flux rates were faster than at lower agonist concentrations (100 and $50 \mu \mathrm{M})$. To directly compare the flux rates between neurites and somata, the somatic flux rate of each cell was plotted against the average of the neuritic fluxes of the same cell in Fig. $4 C$. The black line indicates the identity line, a ratio of 1:1 between somatic and neuritic flux rates. At low somatic fluxes, the neuritic flux rates were consistently more rapid. As the somatic flux rate increased, this difference decreased until the somatic and neuritic flux rates converged. The gray boxes represent cells stimulated with carbachol; the white diamonds display values from experiments in which the RyRs were blocked by the addition of dantrolene. The same trend is observed for both data sets. The results of Figure 4, $A$ and $B$, show that there is a concentrationdependent difference in the ability of carbachol to activate the somatic and the neuritic InsP3-sensitive intracellular calcium stores, which is predominantly determined by the properties of the InsP3-dependent signaling pathway.

To test the possibility that this observation can only be explained by the localized production of InsP3, a membranepermeant form of InsP3 (InsP3-BM) was used to elicit global calcium transients. This analog contains an ester link, which allows the compound to pass into the cell; once inside the cell, the compound is cleaved by an esterase, and free InsP3 is released $(\mathrm{Li}$ et al., 1997, 1998; Thomas et al., 2000). In these experiments, the D-enantiomer of the InsP3-BM was used. Application of this 

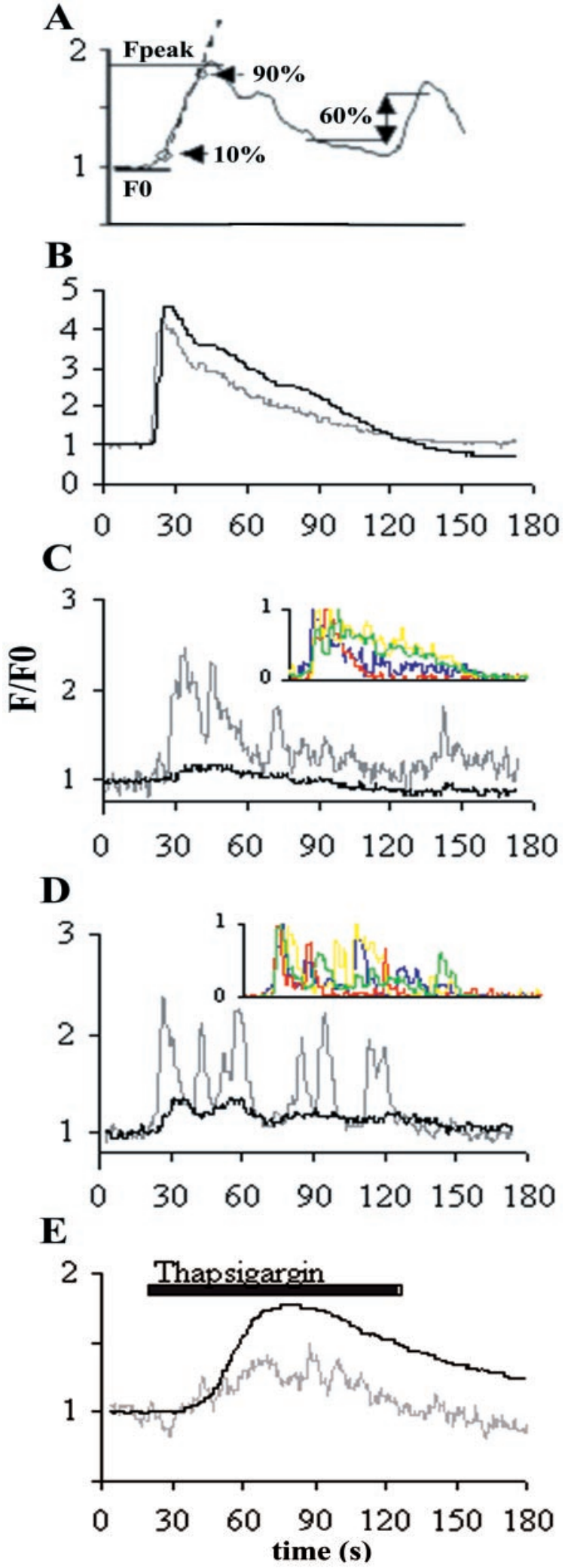

Figure 3. Calcium transients observed in somatic (black lines) and neuritic (gray lines and insets) regions of PC12 cells after carbachol and thapsigargin stimulation. $A$ is a schematic display of the applied analysis pattern (for details, see Materials and Methods). At $500 \mu \mathrm{M}$ carbachol $(B)$, an all-or-nothing response with a steep initial slope and a relatively short duration at half-peak can be observed in both neurites and soma. The temporal delay between the neurite and the soma is very small, and compound $(100 \mu \mathrm{M})$ slowly induced calcium transients in the PC12 cells, in which the rate-limiting step for inducing calcium release is cleavage of the ester bond. This slow cleavage explains the much slower kinetics of the response (Fig. 5) when comparing it with InsP3-induced calcium release mediated by PLC-coupled InsP3 synthesis (Fig. 3B-D). With this analog of InsP3, the diff usion of InsP3 is faster than the buildup of InsP3 at any one site, which results in a uniform InsP3 concentration gradually increasing at a steady rate throughout the cell (Li et al., 1997; Thomas et al., 2000). The transients started in the neurite in 14 of 17 neurites analyzed in 11 different cells, with an average temporal delay of $60.5 \pm 18 \mathrm{sec}$.

Comparison of the magnitude and kinetic properties of the thapsigargin-sensitive pool in somata and neurites

An additional factor modulating the different calcium signaling patterns in neurites and somata is the size of the intracellular calcium stores. Estimates of this factor were obtained by comparing the $F_{\text {peak }}$ and the flux rate of the calcium responses after adding the sarco(endo)plasmic reticulum calcium ATPase pump blocker thapsigargin $(1 \mu \mathrm{M}$ for $120 \mathrm{sec}$ ) (Fig. $3 E)$. The average somatic $F_{\text {peak }}$ of $2.02 \pm 0.13 F / F_{0}(n=8)$ is significantly larger than the average neuritic $F_{\text {peak }}$ of $1.32 \pm 0.08 F / F_{0}(n=13 ; p<$ $0.01)$. The thapsigargin-induced flux rate in the somata $(0.023 \pm$ $\left.0.004 \mathrm{sec}^{-1} ; n=8\right)$ is faster when compared with the neurites $\left(0.014 \pm 0.003 \mathrm{sec}^{-1} ; n=13\right)$. Both parameters imply that the calcium stores are larger in the soma than in the neurites. The higher sensitivity of the neuritic InsP3-sensitive calcium store therefore cannot be explained by a relatively larger intracellular calcium store.

\section{The RyR antagonist dantrolene changes the pattern of somatic and neuritic calcium fluxes at high agonist concentrations}

Although the RyR did not alter the ratio of the flux rates between the soma and neurite in the carbachol-induced calcium signal (Fig. $4 C$ ), there was an obvious change in the distribution pattern of the calcium fluxes in the soma, an effect that was pronounced at high agonist concentrations (Fig. 6). At $500 \mu \mathrm{M}$ carbachol, the calcium signals in the soma were typically large in amplitude, with a rapid flux rate (Figs. $3 B, 6 A$, black traces). After addition of dantrolene, two types of signals were observed (Fig. 6A, gray traces). One was similar to the signal with carbachol alone (top traces), and another appeared as a dampened response with slower flux rates, as observed at lower agonist concentrations (Figs. 3C,D, 6A, bottom gray traces). In Figure 6B, a cumulative distribution plot of the flux rates measured without (black columns) or with (gray columns) dantrolene clearly indicates this

\footnotetext{
there is almost no difference in the slopes and amplitudes of the signals. At $50 \mu \mathrm{M}$ carbachol $(C)$, the somatic response displays a much shallower slope and lower amplitude than the neuritic or the somatic response at 500 $\mu \mathrm{M}$ carbachol. The temporal delay is more pronounced when compared with higher agonist concentrations. The signal is prolonged (neuritic trace and inset in $C$ ), and a fraction of the responses consists of pronounced, but somewhat dampened, repetitive spikes (neuritic trace in $C$ ). The addition of $75 \mu \mathrm{M}$ dantrolene has an enhancing effect on the spiking pattern $(D$ neuritic trace and insets) The insets displayed in $C$ and $D$ are representative neuritic traces from four independent experiments and have been synchronized to the same initiation point and normalized to $F_{\text {peak }}$ to facilitate evaluation of the temporal pattern in the absence and presence of dantrolene at low agonist concentrations. In $E$, a typical trace after stimulation with $100 \mu \mathrm{M}$ thapsigargin is displayed. The somatic response displays a faster calcium flux rate and $F_{\text {peak }}$.
} 
$\mathbf{A}$
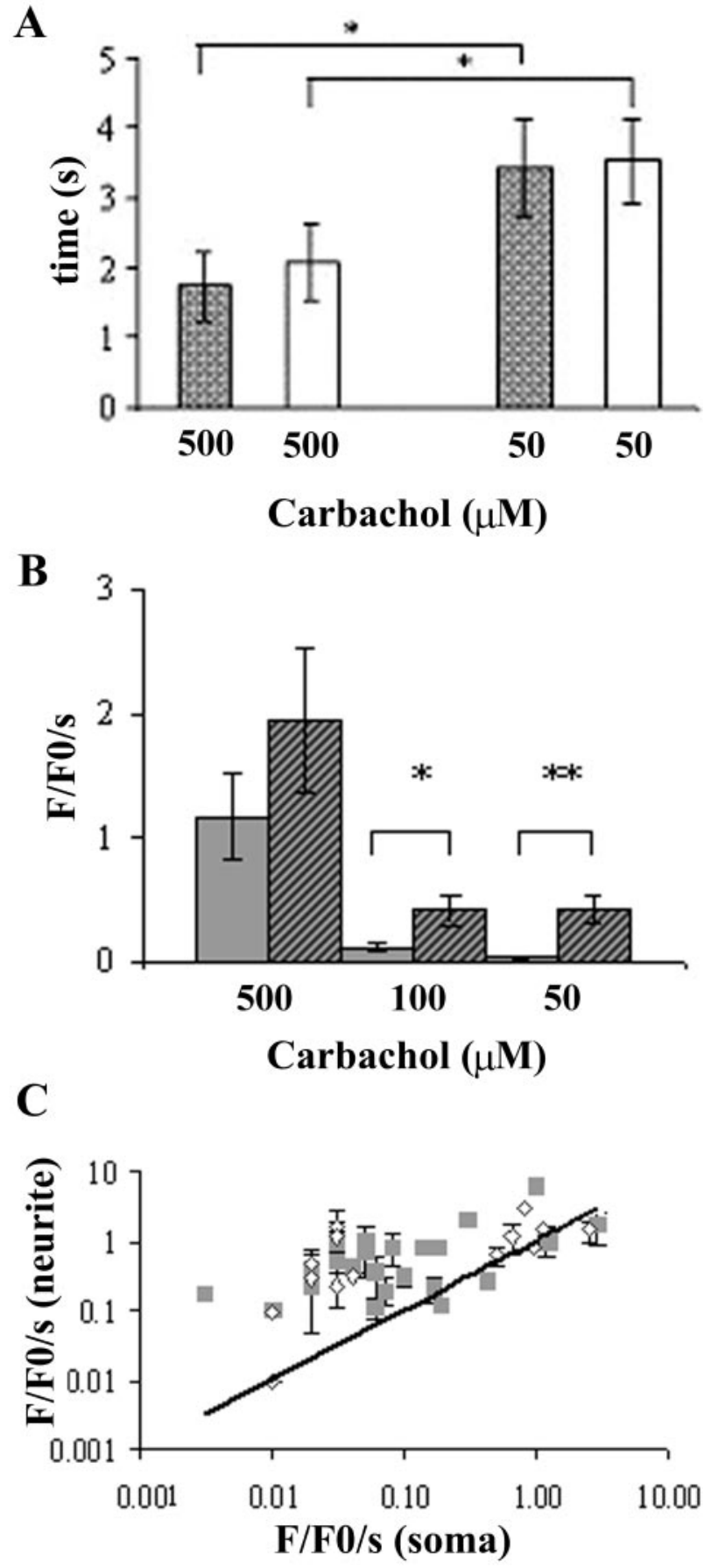

Figure 4. Concentration-dependent differences between neurites and somata in the onset and the flux rate of the calcium signal. In $A$, the delay of the onset of the somatic calcium signal when compared with the neuritic signal is displayed as a function of the carbachol concentration and in the presence (white columns) or absence (gray columns) of $75 \mu \mathrm{M}$ dantrolene. The onset was established as stated in Materials and Methods. At $500 \mu \mathrm{M}$, an average temporal delay of $1.7 \pm 0.5 \mathrm{sec}(n=13)$ between the first neuritic and somatic onset of the calcium rise was detected. Treatment with $50 \mu \mathrm{M}$ carbachol led to a significant increase in this parameter to $3.4 \pm 0.6 \mathrm{sec}(n=19 ; p<0.034)$. A similar trend was seen in the presence of $75 \mu \mathrm{M}$ dantrolene, in which the delay was $2.1 \pm 0.6 \mathrm{sec}$ $(n=12)$ at $500 \mu \mathrm{M}$ carbachol and $3.5 \pm 0.6 \mathrm{sec}$ at $50 \mu \mathrm{M}$ carbachol $(n=$ 14; $p<0.05$ ) (white columns). Asterisks indicate that the differences are statistically significant. In $B$, the calcium flux rates in the somata (gray columns) and neurites (black columns) at different carbachol concentrations are compared with each other. The flux rate is measured as $d(F /$ $\left.F_{0}\right) / d(t)$ over the rising phase of the signal, which is defined in Materials

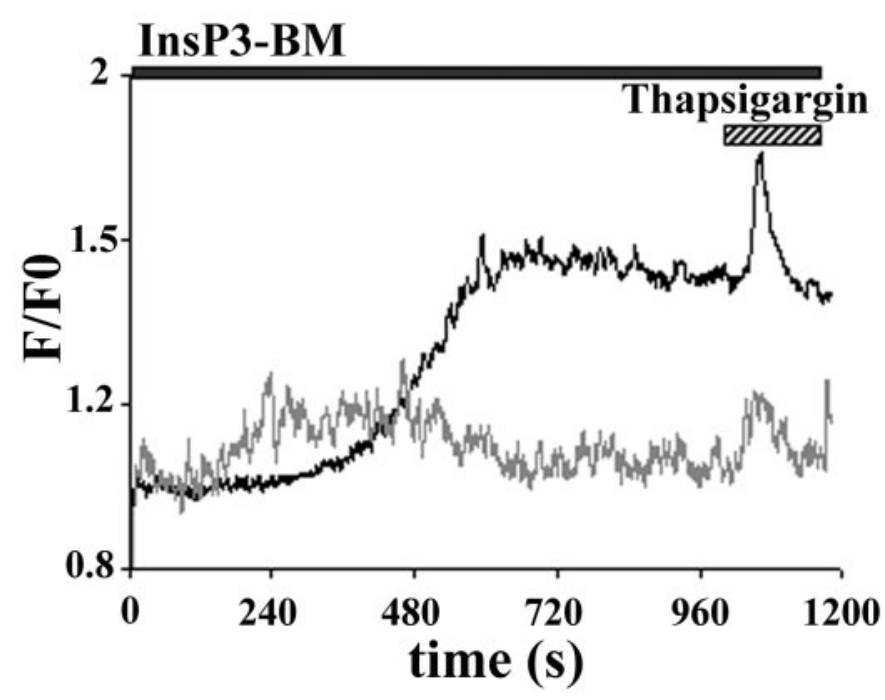

Figure 5. Calcium transients obtained in a neurite and a soma in response to application of InsP3-BM. After addition of InsP3-BM $(100 \mu \mathrm{M})$ the neuritic response ( gray trace) starts before the somatic response (black trace). The small response to $1 \mu \mathrm{M}$ thapsigargin indicates an almost complete depletion of the calcium stores.

difference at $500 \mu \mathrm{M}$ carbachol. After the addition of dantrolene, $50 \%$ of the cells showed somatic flux rates slower than $0.04 \mathrm{sec}^{-1}$, and the other $50 \%$ of the cells responded in the range between 0.5 and $2.5 \mathrm{sec}^{-1}$. This bimodal distribution with a gap of one order of magnitude between the two groups of responses contrasts with the response pattern obtained in the presence of carbachol alone, in which $86 \%$ of the responses were clustered in the interval between 0.3 and $2.9 \mathrm{sec}^{-1}$. Interestingly, this difference was not observed in the neurites, but rather the entire accumulation plot was shifted toward faster flux rates in the absence of dantrolene.

Our morphological studies show that the RyRs are predominantly located in the soma, but weak staining throughout the cell suggests that the RyR is ubiquitously expressed in the cell (Fig. $2 B a, B b)$. Although the levels of RyR may be lower in the neurites than in the soma, inhibition of the RyR effects the spatiotemporal patterning of the calcium signaling in this region of the cell. Two basic temporal organization patterns of the changes of intracellular free calcium can be observed: all or nothing responses (Fig. $3 B, C)$ and repetitive spiking (Fig. $3 D$, neurite and insets). In the soma, none of the measured responses, either with or without

$\leftarrow$

and Methods. The flux rate decreases with lower agonist concentrations. At $500 \mu \mathrm{M}$, the averaged neuritic fluxes are $1.94 \pm 0.58 \mathrm{sec}^{-1}(n=19)$ versus a flux of $1 \pm 0.36 \mathrm{sec}^{-1}(n=7)$ in the somata. Lowering the agonist concentration to $100 \mu \mathrm{M}$ results in statistically significant difference: $0.12 \pm 0.02 \sec ^{-1}(n=8)$ in the soma versus $0.43 \pm 0.12 \sec ^{-1}(n=13)$ in the neurite $(p<0.02)$. An agonist concentration of $50 \mu \mathrm{M}$ results in a significant difference of one order of magnitude: $0.04 \pm 0.01 \mathrm{sec}^{-1}(n=$ $9)$ in the soma versus $0.42 \pm 0.1 \mathrm{sec}^{-1}(n=19)$ in the neurite $(p<0.005)$. In $C$, the average of the neuritic fluxes are plotted as a function of the corresponding somatic fluxes. The gray squares represent cells treated with carbachol only $(n=24)$, and the white diamonds represent cells exposed to carbachol and $75 \mu \mathrm{M}$ dantrolene $(n=15)$. Error bars show the SEM of averaged neuritic fluxes when a cell had more than one neurite. The black line indicates a ratio of 1:1 between neuritic and somatic fluxes. The neuritic fluxes exhibit more rapid kinetics when compared with low somatic fluxes. Increasing the somatic flux, this difference decreases until the somatic and neuritic fluxes increase at the same rate at high somatic fluxes. 
A
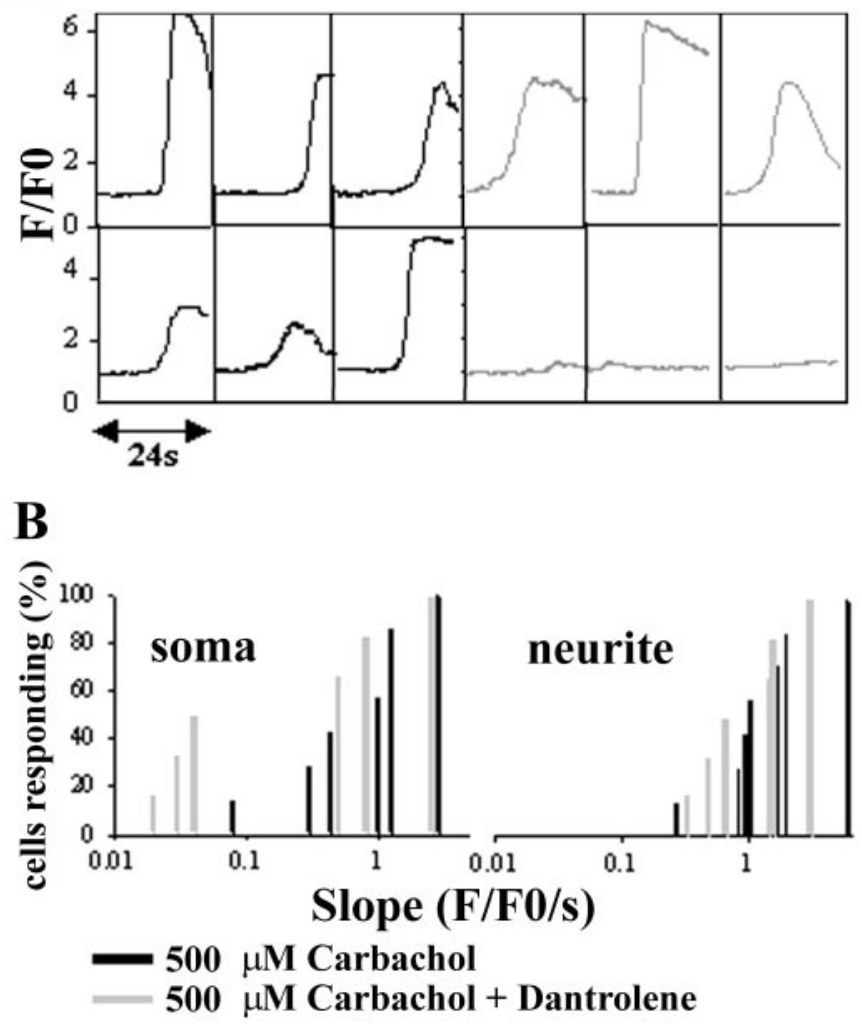

Figure 6. Effects of dantrolene on the distribution of the flux rates in the soma and the neurite. $A$ displays typical traces from somatic ROIs stimulated with $500 \mu \mathrm{M}$ carbachol alone (black traces on the left) and $500 \mu \mathrm{M}$ carbachol when $75 \mu \mathrm{M}$ dantrolene was added (gray traces on the right). All traces are representing different cells in at least five independent experiments. Each box represents a time interval of $24 \mathrm{sec}$, and the $F / F_{0}$ is plotted at the same scale. In $B$, accumulation plots display the distribution of somatic (left) and neuritic (right) fluxes with (gray columns) and without (black columns) dantrolene at $500 \mu \mathrm{M}$ carbachol. Dantrolene $(75$ $\mu \mathrm{M})$ inhibition of RyRs leads to a bimodal distribution of the calcium flux rates: $50 \%$ are slower than $0.04 \mathrm{sec}^{-1}$, and the other $50 \%$ are faster than $0.5 \sec ^{-1}(n=6)$. In contrast, $86 \%$ of cells treated with carbachol alone respond with flux rates faster than $0.3 \mathrm{sec}^{-1}(n=7)$. This effect cannot be observed in the neurites; the addition of dantrolene only leads to a small shift toward slower flux rates.

Table 1. Percentage of neurites exhibiting repetitive spiking patterns

\begin{tabular}{llr} 
Carbachol $(\mu \mathrm{M})$ & $(+)$ Dantrolene & $(-)$ Dantrolene \\
\hline 500 & $27 \%(n=12)$ & $0 \%(n=13)$ \\
50 & $67 \%(n=15)$ & $21 \%(n=15)$
\end{tabular}

Dantrolene increases the probability of repetitive spiking in neurites. The percentage of neurites exhibiting repetitive spiking patterns of the calcium response at 500 and $50 \mu \mathrm{M}$ carbachol in the absence of dantrolene (left column) increases when dantrolene is added (right column).

dantrolene $(n=39)$, fulfilled our defined criterion for a repetitive spike, as described in Materials and Methods. In contrast, repetitive spiking could be evoked in the neurites by low agonist concentrations. RyR inhibition with dantrolene increased the probability of repetitive spiking in the neurites (Table 1; Fig. $3 C, D$, neuritic trace and insets).

\section{DISCUSSION}

Neuronally differentiated PC12 cells show the basic functional and morphological properties of peripheral sympathetic ganglion cells in primary culture, with respect to excitability, secretion, and expression of metabotropic and ionotropic receptors (Greene and Tischler, 1976; Koizumi et al., 1999). In the adult brain, the InsP3R type I is the main functional isoform, the levels of InsP3R type III are low, and those of InsP3R type II are negligible (Blackshaw et al., 2000). However, in dorsal root ganglion cells, as well as in adult rat hippocampus, initiation of programmed cell death leads to the upregulation of InsP3R type III and can be prevented by antisense oligonucleotides to InsP3R type III in dorsal root ganglion cells (Blackshaw et al., 2000). Consequently, studying the interaction of the InsP3R isoforms types I and III and RyRs in the PC12 cell line renders important insights into mechanisms of differential quantitative, spatial, and temporal patterning of calcium signals in neurons.

The experimentally observed concentration-dependent temporal delay between neuritic and somatic calcium signals, as well as the differences in neuritic and somatic flux rates, indicate that neurites have a lower activation threshold for the initiation and propagation of InsP3-mediated calcium signals than somata. Differences in the metabotropic receptor distribution and the surface-to-volume ratio of the different compartments have been proposed as the mechanisms underlying these phenomena (Lorenzon et al., 1995; Fink et al., 2000; van Acker et al., 2000). Results presented here imply the need for additional explanations.

The distribution of mAChRs in PC12 cells (Fig. 2Ca,Cc) clearly indicates that the majority of PLC-mediated InsP3 generation takes place in the soma, a mechanism that compensates for differences in surface-to-volume ratio. Nevertheless, even small clusters of mAChRs might be more efficient at producing high focal InsP3 concentrations in neurites (Fig. 2Cc, white arrows) considering the comparatively smaller volume into which InsP3 molecules diff use. To test the possibility that focal gradients of the InsP3 concentration can account for differences in the observed temporal difference in calcium signaling, we generated a mathematical model using the Virtual Cell Programming Platform [http://www.nrcam.uchc.edu/ (Center for Biomedical Imaging Technology, University of Connecticut, Farmington, CT)]. The geometry of a generic PC12 cell was represented by a spherical cell body (diameter of $20 \mu \mathrm{m}$ ) with a single neurite [50-100 $\mu \mathrm{m}$ long, $2 \mu \mathrm{m}$ diameter (Reber and Schindelholz, 1996)] (Fig. 7A). The resting calcium concentration was $20 \mathrm{~nm}$, the resting InsP3 concentration was $10 \mathrm{nM}$, InsP3 diffusion was $283 \mu \mathrm{m}^{2} / \mathrm{sec}$, and half-time for InsP3 degradation was $1 \mathrm{sec}$ (Allbritton et al., 1992). With the additional assumption of a constant release of InsP3 at a single focal site at a fixed rate and that the threshold for InsP3R activation is $22 \mathrm{~nm}$ for InsP3R type I and $94 \mathrm{~nm}$ for InsP3R type III, the InsP3 concentration in the center of the soma was calculated as a function of the rate of InsP3 production (Fig. $7 A, B$ ). The values for the threshold of activation for the InsP3R were chosen using the $\mathrm{EC}_{50}$ values for activation of calcium release measured in intact cells (Wojcikiewicz and Luo, 1998). By using the $\mathrm{EC}_{50}$ as threshold values, the time needed to activate calcium release was overestimated. At the rate of InsP3 production measured in oocytes $\left[2 \mathrm{~mm} \cdot \mu \mathrm{m}^{-1} \cdot \mathrm{sec}^{-1}\right.$ (Allbritton et al., 1992)], the InsP3 concentration in the center of the soma exceeds threshold for InsP3R type I within $5 \mathrm{sec}$ (Fig. $7 B$ ). Because InsP3 is produced only at the tip of the neurite in this simulation (between 40 and $50 \mu \mathrm{M}$ from the neurite-soma junction), the concentration of InsP3 in the neurite is significantly higher than in the soma (Fig. $7 A$ ); at the junction between the neurite and soma, the InsP3 concentration would be $23 \mathrm{~nm}$ in $<1.5 \mathrm{sec}$. The time-to-threshold 


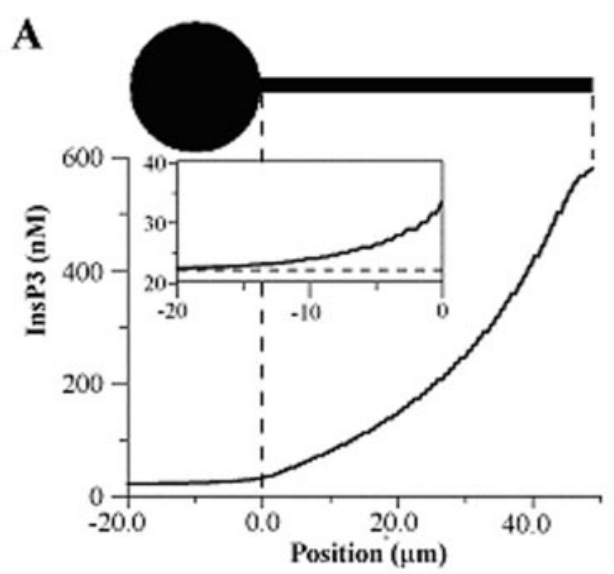

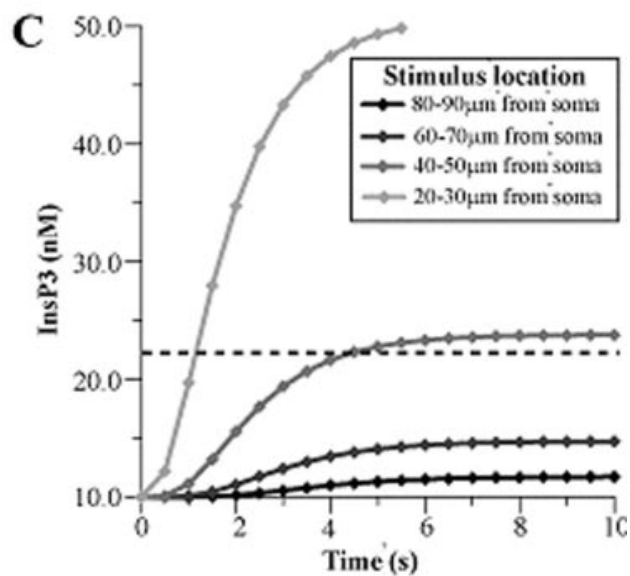

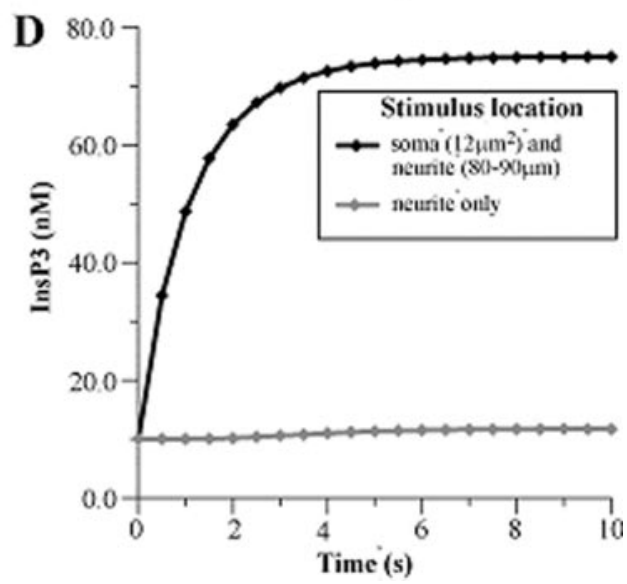

Figure 7. Model of InsP3 diffusion in PC12 cells. $A$ shows the model of the neuron with the profile of the InsP3 concentration calculated after $10 \mathrm{sec}$ of InsP 3 production in the neurite at $40-50$ $\mu \mathrm{m}$ from the soma at a rate of 2 $\mathrm{mm} \cdot \mu \mathrm{m}^{-1} \cdot \mathrm{sec}^{-1}$. The inset shows the region of the soma, in which $-10 \mu \mathrm{m}$ is the center of the soma. Note that the InsP3 concentration is higher than 22 $\mathrm{nM}$, the threshold for the InsP3R type I at all locations. $B$, The effect of altering the rate of InsP3 production at a fixed location in the neurite $(40-50 \mu \mathrm{m}$ from the soma) is calculated. $C$, The effect of altering the location of InsP3 production in the neurite is calculated. In $D$, an additional site of InsP3 production in the soma is added to the subthreshold stimulus at the end of a very long neurite. Note that the threshold for InsP3R activation is achieved within $0.5 \mathrm{sec}$ for the InsP3R type I and within $4 \mathrm{sec}$ for the InsP3R type III. in the center of the soma is reduced to $1 \mathrm{sec}$ when the rate of InsP3 production is increased to $10 \mathrm{~mm} \cdot \mu \mathrm{m}^{-1} \cdot \mathrm{sec}^{-1}$ (Fig. $7 B$ ). If the concentration of InsP3 in the center of the soma is calculated as a function of the stimulus location and the site of production is moved closer to the soma, then the threshold for the InsP3R type I is achieved more quickly (Fig. $7 C$ ). The addition of a single focal site of InsP3 production in the soma reduces the time to reach threshold for both InsP3R type I and III to $<0.25$ sec (Fig. 7D). In the experiments presented in this paper, it is assumed that InsP3 will be produced by mAChRs, which are found on the plasma membrane of the soma and neurite (Fig. 2C). Thus, the simulations support the assumption that the concentration of InsP3 will exceed threshold uniformly throughout the cell within $1 \mathrm{sec}$, which is faster than the observed temporal delay in the initiation of the calcium transient in neurite and soma. Therefore, local differences in the concentration of InsP3 cannot explain the temporal delay.

To achieve an even elevation of InsP3 within the cell, the cell-permeant ester of InsP3 (InsP3-BM) was used. Using this compound, InsP3 diff usion is much faster than InsP3 production, and local differences cannot develop. As shown in the model (Fig. 7), production of InsP3 at only one site in the soma allows rapid accumulation of InsP3 throughout the soma, suggesting that, even with a lower rate of production of InsP3 from the ester, there is a much larger region of InsP3 production, which will rapidly lead to equilibration of the InsP3 concentration. Consequently, the temporal difference observed when using InsP3-BM ester must be attributable to factor(s) downstream of InsP3 production, rendering neuritic InsP3Rs more sensitive to InsP3 than somatic InsP3Rs.
This factor still needs to be determined. Chromogranins have been shown to enhance the effect of low InsP3 concentrations on InsP3Rs in planar lipid bilayers (Thrower et al., 2002), and this protein is found predominantly in the neurites (Fig. 2D). However, there are many other molecules that might interact with the InsP3R in the neurites that would modulate its sensitivity to InsP3 (e.g., calmodulin and FK506-binding protein), but their subcellular distribution appears to be uniform in PC12 cells (data not shown). Another option is the effect of a larger calcium store in the neurite rendering neuritic InsP3Rs more sensitive. However, our experiments with thapsigargin show that the neuritic calcium store is smaller.

The exclusive expression in the soma of InsP3R type III, which is less sensitive to InsP3 than the other InsP3R isoforms, might be an explanation for the observed differences in calcium signaling between soma and neurites. PC12 cells, however, express both InsP3R types I and III in the soma. Consequently, to actually account for the temporal difference in initial signal initiation, one would have to propose the formation of heterotetramers in which gating is determined by the subtype with the lowest affinity for InsP3. Another possible explanation is a much lower density of InsP3R type I compared with InsP3R type III. However, InsP3R types I and III have been shown to be evenly distributed in the perinuclear signal initiation region in HeLa cells (Thomas et al., 2000). It seems more likely that the differential distribution of InsP3R types I and III creates an additional InsP3-dependent pool with a lower affinity for InsP3 and higher activation threshold that determines the magnitude, but not the initiation, of the signal in the soma.

In this context, a functional role of RyRs in the soma is implied 
by the change in the distribution of the slopes of the calcium transients in the presence of dantrolene. Regarding the use of dantrolene as a pharmacological tool to inhibit ryanodine receptors, a direct interaction between dantrolene and RyRs has only been shown for subtypes 1 and 3 at low concentrations of dantrolene $(10 \mu \mathrm{M})$ (Zhao et al., 2001). Nevertheless, higher concentrations of dantrolene inhibit RyR type 2-dependent calcium release in neuronal and cardiac preparations (O’Mara et al., 1995; Nelson et al., 1999; Usachev and Thayer, 1999; Yu et al., 2000). The advantage of using this compound over previously used protocols for blocking RyRs [caffeine-ryanodine pretreatment (Reber and Schindelholz, 1996; Bennett et al., 1998)] is that there is no depletion of the endoplasmic reticulum calcium store. RyRs do not appear to have an impact on the initiation of calcium signals (Fig. 4A), but rather they play a modulatory role in shaping the temporospatial pattern of the signal, once the signal is initiated. After the RyRs are inhibited, the somatic InsP3sensitive calcium store is divided into two distinct pools. They could be molecularly distinguished by the low-threshold InsP3 sensor InsP3R type I and the high-threshold InsP3 sensor InsP3R type III. Therefore, the probability for all-or-nothing calcium release from the InsP3R type III-sensitive pool (Hagar et al., 1998) is reduced when blocking RyRs because the additional calcium release via RyRs is needed to increase the probability for InsP3R type III activation (Hagar et al., 1998; Mak et al., 2001). This is reflected by the bimodal distribution of the slopes after RyR block (Fig. 6). In the neurites, there is only a small decrease in the magnitude of the slopes when dantrolene is added, indicating the absence of a second, high-threshold InsP3-dependent pool attributable to the lack of InsP3R type III.

Dantrolene inhibition of RyRs dramatically increases the probability of neuritic spiking at all given agonist concentrations. Consequently, RyRs appear to override the oscillatory responses mediated by InsP3Rs. The absence of spikes in the soma can be explained by the increase in calcium ATPases in the plasma membrane during the differentiation process of PC12 cells (Keller and Grover, 2000). The RyR is inactivated at a much higher calcium concentration than the InsP3R type I [1 vs $>100 \mu \mathrm{M}$ in single channel measurements (Bezprozvanny et al., 1991; Bootman et al., 2001)]. Inactivation of the InsP3R by high intracellular free calcium with subsequent reactivation after removal of calcium from the cytosol and relatively slower recovery of the InsP3R from inactivation are necessary factors determining oscillatory response patterns (Bezprozvanny and Ehrlich, 1994; Hajnoczky and Thomas, 1994, 1997). In the experiments presented here, the negative feedback effect of intracellular free calcium on InsP3R type I loses its impact on the calcium signal because it is overridden by high intracellular calcium concentrations produced by RyR-mediated calcium-induced calcium release in the neurites.

\section{Conclusion}

Global calcium signals in neurites modify information processing by affecting synaptic strength and excitability. In the soma, more fundamental changes at the transcription level are mediated by elevations in global calcium signals (Berridge, 1998; Usachev and Thayer, 1999). Thus, different sensitivities of the InsP3Rs in the neurites and somata provide a mechanism of signal integration. Short-term changes in neuronal function in the neurite can be discriminated from long-term changes on the protein synthesis level as a function of stimulus strength. In summary, the InsP3R type I with its lower activation is well suited for short-term changes in intracellular calcium. Its low InsP3 sensitivity (Hagar and Ehrlich, 2000) makes the InsP3R type III the ideal gatekeeper for high-amplitude somatic calcium surges, which need to be carefully controlled. The aforementioned impact of InsP3R type III on apoptotic cell death correlates with the proposed role of a high-threshold gatekeeper.

Based on these predictions, RyRs add a new level of complexity to intracellular calcium dynamics. Being activated at calcium concentrations $>1 \mu \mathrm{M}$ (Bezprozvanny et al., 1991; Bootman et al., 2001), the RyR start modifying the intracellular calcium signal after initiation of the signal via InsP3Rs, which explains why no impact on signal initiation was observed in our experiments. Based on the geometric and molecular environment, the RyRs differentially affect the quantitative and qualitative aspects of the calcium signal in distinct regions of the cell. In the soma, they serve as a molecular switch, facilitating the recruitment of additional calcium, shaping the calcium flux rate by adjusting the sensitivity of the low-affinity InsP3R type III, and releasing calcium themselves. In the neurites, RyRs determine the probability of repetitive spiking. These roles of the RyR add an additional level of complexity to coincidence detection of intracellular calcium signals. This concept of a compartment-specific distribution of ion channels is analogous to the finding in the plasma membrane of pyramidal neurons, in which low-threshold voltage-gated calcium channels are found in the distal dendrite and highthreshold voltage-gated calcium channels are refined to the apical dendrite and soma of the cell (Christie et al., 1995).

\section{REFERENCES}

Allbritton NL, Meyer T, Stryer L (1992) Range of messenger action of calcium ion, inositol 1,4,5-trisphosphate. Science 258:1812-1815.

Bennett DL, Bootman MD, Berridge MJ, Cheek TR (1998) $\mathrm{Ca}^{2+}$ entry into PC12 cells initiated by ryanodine receptors or inositol $1,4,5$ trisphosphate receptors. Biochem J 329:349-357.

Berkeley JL, Levey AI (2000) Muscarinic activation of mitogenactivated protein kinase in PC12 cells. J Neurochem 75:487-493.

Berridge MJ (1998) Neuronal calcium signaling. Neuron 21:13-26.

Bezprozvanny I, Ehrlich B (1994) Inositol (1,4,5)-trisphosphate gated Ca channels from canine cerebellum: divalent cation conduction properties and regulation by intraluminal Ca. J Gen Physiol 104:821-856.

Bezprozvanny I, Watras J, Ehrlich BE (1991) Bell-shaped calciumresponse curves of Ins $(1,4,5) \mathrm{P} 3$ - and calcium-gated channels from endoplasmic reticulum of cerebellum. Nature 351:751-754.

Blackshaw S, Sawa A, Sharp AH, Ross CA, Snyder SH, Khan AA (2000) Type 3 inositol 1,4,5-trisphosphate receptor modulates cell death. FASEB J 14:1375-1379.

Bootman MD, Collins TJ, Peppiatt CM, Prothero LS, MacKenzie L, De Smet P, Travers M, Tovey SC, Seo JT, Berridge MJ, Ciccolini F, Lipp P (2001) Calcium signalling-an overview. Semin Cell Dev Biol $12: 3-10$

Christie BR, Eliot LS, Ito K, Miyakawa H, Johnston D (1995) Different $\mathrm{Ca}^{2+}$ channels in soma and dendrites of hippocampal pyramidal neurons mediate spike-induced $\mathrm{Ca}^{2+}$ influx. J Neurophysiol 73:2553-2557.

Ehrlich BE, Kaftan E, Bezprozvannaya S, Bezprozvanny I (1994) The pharmacology of intracellular $\mathrm{Ca}^{2+}$-release channels. Trends Pharmacol 15:145-149.

Fink CC, Slepchenko B, Moraru II, Watras J, Schaff JC, Loew LM (2000) An image-based model of calcium waves in differentiated neuroblastoma cells. Biophys J 79:163-183.

Ghosh A, Greenberg ME (1995) Calcium signaling in neurons: molecular mechanisms and cellular consequences. Science 268:239-247.

Giannini G, Conti A, Mammarella S, Scrobogna M, Sorrentino V (1995) The ryanodine receptor/calcium channel genes are widely and differentially expressed in murine brain and peripheral tissues. J Cell Biol 128:893-904.

Greene LA, Tischler AS (1976) Establishment of a noradrenergic clonal line of rat adrenal pheochromocytoma cells which respond to nerve growth factor. Proc Natl Acad Sci USA 73:2424-2428.

Hagar R, Burgstahler A, Nathanson M, Ehrlich B (1998) Type III InsP3 receptors stay open in the presence of increased calcium. Nature 396:81-84.

Hagar RE, Ehrlich BE (2000) Regulation of the type III InsP(3) receptor by InsP(3) and ATP. Biophys J 79:271-278. 
Hajnoczky G, Thomas AP (1994) The inositol trisphosphate calcium channel is inactivated by inositol trisphosphate. Nature 370:474-477.

Hajnoczky G, Thomas AP (1997) Minimal requirements for calcium oscillations driven by the IP3 receptor. EMBO J 16:3533-3543.

Hirata K, Nathanson M, Sears M (1998) Novel paracrine signaling mechanism in the ocular ciliary bilayer epithelium. Proc Natl Acad Sci USA 95:8381-8386.

Hirata K, Nathanson MH, Burgstahler AD, Okazaki K, Mattei E, Sears ML (1999) Relationship between inositol 1,4,5-trisphosphate receptor isoforms and subcellular $\mathrm{Ca}^{2+}$ signaling patterns in nonpigmented ciliary epithelia. Invest Ophthalmol Vis Sci 40:2046-2053.

Hirata K, Pusl T, O’Neill A, Dranoff JA, Nathanson MH (2002) The type II inositol 1,4,5-trisphosphate receptor can trigger $\mathrm{Ca}^{2+}$ waves in rat hepatocytes. Gastroenterology 122:1088-1100.

Jaffe DB, Brown TH (1994) Metabotropic glutamate receptor activation induces calcium waves within hippocampal dendrites. J Neurophysiol 72:471-474.

Keller D, Grover AK (2000) Nerve growth factor treatment alters $\mathrm{Ca}^{2+}$ pump levels in PC12 cells. NeuroReport 11:65-68.

Koizumi S, Bootman MD, Bobanovic LK, Schell MJ, Berridge MJ, Lipp $\mathrm{P}$ (1999) Characterization of elementary $\mathrm{Ca}^{2+}$ release signals in NGF-differentiated PC12 cells and hippocampal neurons. Neuron 22:125-137.

Li W, Schultz C, Llopis J, Tsien RY (1997) Membrane-permeant esters of inositol polyphosphates, chemical syntheses and biological applications. Tetrahedron 53:12017-12040.

Li W, Llopis J, Whitney M, Zlokarnik G, Tsien RY (1998) Cellpermeant caged InsP3 ester shows that $\mathrm{Ca}^{2+}$ spike frequency can optimize gene expression. Nature 392:936-941.

Lorenzon P, Zacchetti D, Codazzi F, Fumagalli G, Meldolesi J, Grohovaz F'(1995) $\mathrm{Ca}^{2+}$ waves in PC12 neurites: a bidirectional, receptor-oriented form of $\mathrm{Ca}^{2+}$ signaling. J Cell Biol 129:797-804.

MacKrill JJ (1999) Protein-protein interactions in intracellular $\mathrm{Ca}^{2+}$ release channel function. Biochem J 337:345-361.

Mak DO, McBride S, Raghuram V, Yue Y, Joseph SK, Foskett JK (2000) Single-channel properties in endoplasmic reticulum membrane of recombinant type 3 inositol trisphosphate receptor. J Gen Physiol 115:241-256.

Mak DO, McBride S, Foskett JK (2001) Regulation by $\mathrm{Ca}^{2+}$ and inositol 1,4,5-trisphosphate (InsP3) of single recombinant type 3 InsP3 receptor channels. $\mathrm{Ca}^{2+}$ activation uniquely distinguishes types 1 and 3 insp3 receptors. J Gen Physiol 117:435-446.

Maravall M, Mainen ZF, Sabatini BL, Svoboda K (2000) Estimating intracellular calcium concentrations and buffering without wavelength ratioing. Biophys J 78:2655-2667.

Marx SO, Reiken S, Hisamatsu Y, Gaburjakova M, Gaburjakova J, Yang YM, Rosemblit N, Marks AR (2001) Phosphorylation-dependent regulation of ryanodine receptors: a novel role for leucine/isoleucine zippers. J Cell Biol 153:699-708.

Mignery G, Sudhof TC, Takei K, De Camilli P (1989) Putative receptor for inositol 1,4,5-trisphosphate similar to ryanodine receptor. Nature 342:192-195.

Nakamura T, Barbara JG, Nakamura K, Ross WN (1999) Synergistic release of $\mathrm{Ca}^{2+}$ from IP3-sensitive stores evoked by synaptic activation of mGluRs paired with backpropagating action potentials. Neuron 24:727-737.

Nakamura T, Nakamura K, Lasser-Ross N, Barbara JG, Sandler VM,
Ross WN (2000) Inositol 1,4,5-trisphosphate (IP3)-mediated $\mathrm{Ca}^{2+}$ release evoked by metabotropic agonists and backpropagating action potentials in hippocampal CA1 pyramidal neurons. J Neurosci 20:8365-8376.

Ndoye A, Buchli R, Greenberg B, Nguyen VT, Zia S, Rodriguez JG, Webber RJ, Lawry MA, Grando SA (1998) Identification and mapping of keratinocyte muscarinic acetylcholine receptor subtypes in human epidermis. J Invest Dermatol 111:410-416.

Nelson TJ, Zhao WQ, Yuan S, Favit A, Pozzo-Miller L, Alkon DL (1999) Calexcitin interaction with neuronal ryanodine receptors. Biochem J 341:423-433.

Ogden D, Capiod T (1997) Regulation of $\mathrm{Ca}^{2+}$ release by InsP3 in single guinea pig hepatocytes and rat Purkinje neurons. J Gen Physiol 109:741-756.

O’Mara SM, Rowan MJ, Anwyl R (1995) Dantrolene inhibits long-term depression and depotentiation of synaptic transmission in the rat dentate gyrus. Neuroscience 68:621-624.

Patel S, Joseph SK, Thomas AP (1999) Molecular properties of inositol 1,4,5-trisphosphate receptors. Cell Calcium 25:247-264.

Perez PJ, Ramos-Franco J, Fill M, Mignery GA (1997) Identification and functional reconstitution of the type 2 inositol 1,4,5-trisphosphate receptor from ventricular cardiac myocytes. J Biol Chem 272:23961-23969.

Reber BF, Schindelholz B (1996) Detection of a trigger zone of bradykinin-induced fast calcium waves in PC12 neurites. Pflügers Arch 432:893-903.

Rizzuto R (2001) Intracellular $\mathrm{Ca}^{2+}$ pools in neuronal signalling. Curr Opin Neurobiol 11:306-311.

Sitsapesan R, Williams A 1998 The structure and function of ryanodine receptors. London: Imperial College.

Thomas D, Lipp P, Tovey SC, Berridge MJ, Li W, Tsien RY, Bootman MD (2000) Microscopic properties of elementary $\mathrm{Ca}^{2+}$ release sites in non- excitable cells. Curr Biol 10:8-15.

Thrower E, Park H, Seung H, Yoo S, Ehrlich B (2002) Effects of chromogranins on InsP3-gated channel activity. J Biol Chem 277:1580115806.

Usachev YM, Thayer SA (1999) Controlling the urge for a $\mathrm{Ca}^{2+}$ surge: all-or-none $\mathrm{Ca}^{2+}$ release in neurons. BioEssays 21:743-750.

van Acker K, Bautmans B, Bultynck G, Maes K, Weidema AF, de Smet P, Parys JB, de Smedt H, Missiaen L, Callewaert G (2000) Mapping of IP(3)-mediated $\mathrm{Ca}^{2+}$ signals in single human neuroblastoma SH-SY5Y cells: cell volume shaping the $\mathrm{Ca}^{2+}$ signal. $\mathrm{J}$ Neurophysiol 83:1052-1057

Wojcikiewicz RJ, Luo SG (1998) Differences among type I, II, and III inositol-1,4,5-trisphosphate receptors in ligand-binding affinity influence the sensitivity of calcium stores to inositol-1,4,5-trisphosphate. Mol Pharmacol 53:656-662.

Wojcikiewicz RJH (1995) Type I, II, III inositol 1,4,5-trisphosphate receptors are unequally susceptible to down-regulation and are expressed in markedly different proportions in different cell types. J Biol Chem 270:11678-11683.

Yu G, Zucchi R, Ronca-Testoni S, Ronca G (2000) Protection of ischemic rat heart by dantrolene, an antagonist of the sarcoplasmic reticulum calcium release channel. Basic Res Cardiol 95:137-143.

Zhao F, Li P, Chen SR, Louis CF, Fruen BR (2001) Dantrolene inhibition of ryanodine receptor $\mathrm{Ca}^{2+}$ release channels. Molecular mechanism and isoform selectivity. J Biol Chem 276:13810-13816. 